\title{
Diversité Des Loranthaceae Et Leur Impact Sur Vitellaria Paradoxa C.F.Gaertn.: Un Fruitier À Grande Valeur Socio-Économique Au Nord-Bénin
}

\author{
Innocent D. Y. Ahamidé \\ Monique G. Tossou \\ Hounnankpon Yédomonhan \\ Aristide C. Adomou
}

Laboratoire de Botanique et Ecologie végétale, Faculté des Sciences et

Techniques, Université d'Abomey-Calavi, Cotonou, Bénin.

\section{Janvier Houénon}

Département du Génie de l'Environnement, Ecole Polytechnique d'AbomeyCalavi, Université d'Abomey-Calavi, Bénin.

\section{Akpovi Akoègninou}

Laboratoire de Botanique et Ecologie végétale, Faculté des Sciences et

Techniques, Université d'Abomey-Calavi, Cotonou, Bénin

doi: 10.19044/esj.2017.v13n24p217 URL:http://dx.doi.org/10.19044/esj.2017.v13n24p217

\begin{abstract}
Shea butter: Vitellaria paradoxa C. F. Gaertn (Sapotaceae) is a fruit tree in the Sudano-Sahelian region where it is a major economic source. Unfortunately, in Benin, the species has been revealed in recent decades, the target of parasitic vascular plants of Loranthaceae family that threaten its conservation. The present study, carried out in northern Benin, evaluated the impact of these parasitic plants on the shea of two phytogeographical zones. The botanical inventory identified three species of Loranthaceae in fields and protected areas. The rate of infestation and parasite density were assessed and their variation on shea was assessed. The results show that shea is parasitized by three species of Loranthaceae in varying proportions: Agelanthus dodoneifolius (DC.) Polh. and Wiens (191.75 tufts / ha), Tapinanthus globiferus (A. Rich.) Van Tieghem (70.57 tufts / ha) and T. Ophiodes (Sprague) (2 tufts / ha). The impact of Loranthaceae on shea productivity varies significantly between fields and protected areas with infestation rates of $87 \%$ versus $42 \%$ and average densities per shea foot of 14.76 tufts versus 3.62 tufts. These results are data to be taken into account in the control programs against Loranthaceae which parasitize shea.
\end{abstract}


Keywords: Shea - Loranthaceae - infestation rate - parasitic density - control programs - Benin

\section{Résumé}

Le karité : Vitellaria paradoxa C. F. Gaertn (Sapotaceae) est un arbre fruitier de la région soudano-sahélienne où l'essence constitue une grande source économique. Malheureusement, au Benin, l'espèce s'est révélée dans ces dernières décennies, la cible des plantes vasculaires parasites de la famille des Loranthaceae qui menacent sa conservation. La présente étude, réalisée au Nord du Bénin a permis d'évaluer l'impact de ces plantes parasites sur le karité de deux zones phytogéographiques. L'inventaire botanique a permis de recenser trois espèces de Loranthaceae dans les champs et les aires protégées. Le taux d'infestation et la densité parasitaire ont été évalués et leur variation sur le karité a été appréciée. Les résultats révèlent que le karité est parasité par trois espèces de Loranthaceae dans des proportions variées : Agelanthus dodoneifolius (DC.) Polh. et Wiens (191,75 touffes/ha), Tapinanthus globiferus (A. Rich.) Van Tieghem (70,57 touffes/ha) et $T$. Ophiodes (Sprague) (2 touffes/ha). L'impact des Loranthaceae sur le karité varie significativement entre les champs et les aires protégées avec respectivement des taux d'infestation de $87 \%$ contre 42 $\%$ et des densités moyennes par pied de karité de 14,76 touffes contre 3,62 touffes. Ces résultats obtenus constituent des données dont il faut tenir compte dans les programmes de lutte contre les Loranthaceae qui parasitent le karité.

Mots-clés : Karité - Loranthaceae - taux d'infestation - densité parasitaire programmes de lutte - Benin

\section{Introduction}

Au Bénin, les arbres et arbustes fruitiers grâce aux multiples produits forestiers non ligneux qu'ils fournissent, présentent des intérêts alimentaire, médicinal et économique certains pour les populations qui contribuent à la gestion durable des écosystèmes (Eyog Matig et al., 2002). Le karité : Vitellaria paradoxa C.F.Gaertn. (Sapotaceae), arbre fruitier de la région sudano-sahélienne; est une espèce endémique caractéristique de la flore ligneuse des savanes boisées d'Afrique, exploitée dans son intégralité (White, 1983). Elle est par ailleurs signalée comme la deuxième sur la liste des 10 espèces ligneuses alimentaires prioritaires pour le Bénin (Eyog Matig et al., 2002). La priorité accordée à cet arbre fruitier est liée à son importance économique considérable. Les fortes pressions exercées sur les vergers à karités riquent de provoquer leur extinction (Eyog Matig et al., 2002). En effet, les estimations du Ministère de l'Agriculture de l'Elevage et de la 
Pêche au Bénin en 2008 rapportent que le karité constitue le troisième produit d'exportation après le coton et l'anacarde. Au plan national, ce même auteur rapporte que la part annuelle des revenues des exportations liées au karité s'élève à 1,05 milliards de francs CFA. Le fruit du karité est consommé par les populations tant rurales qu'urbaines. Selon Wiesman et Maranz (2001), la pulpe des fruits du karité contient de grandes quantités de protéines, de minéraux et ses amandes sont riches en acides gras notamment en acides oléique, stéarique, linoléique et palmitique. Le beurre de karité est consommé localement et exploité en cosmétique, confiserie, pâtisserie, ainsi que dans la fabrication du chocolat et de margarines végétales (Gnanglè, 2005). Les produits issus du karité sont utilisés par les tradipraticiens de la Côte d'Ivoire dans le traitement de 43 affections en moyenne (Diarassouba $e t$ al., 2008). L'arbre est aussi impliqué dans l'amélioration du microclimat et de la fertilité du sol pour les cultures associées (Bayala et al., 2002).

Malheureusement, aujourd'hui, la productivité et la conservation de l'arbre à karité sont limitées par plusieurs facteurs notamment les pressions anthropiques en l'occurrence, l'abattage des arbres pour le bois de feu, les feux de brousse et l'agriculture en général (cotonculture). A ces facteurs anthropiques, s'ajoutent ceux naturels liés à la sénescence de la plupart des individus de karité, le faible pouvoir germinatif des graines et les difficultés liées à la pollinisation des fleurs (Sanou et Lamien, 2011). De plus, les peuplements de karités sont attaqués par les plantes vasculaires parasites de la famille des Loranthaceae. Ces parasites sans être spécifiques au karité, ont trouvé en lui un hôte favorable (Soro, 1999). Ces hémiparasites, une fois implantés sur leurs hôtes affaiblissent ces derniers, réduisent de façon drastique leur productivité fruitière et menacent leur statut de conservation (Houéhanou et al., 2011 ; Edagbo et al., 2013). Cette attaque du karité par les Loranthaceae a été déjà signalée au Burkina Faso où $95 \%$ des individus sont parasités (Boussim et al., 1993). Face à cette situation si préoccupante, Boussim et Médah (2004) ont testé plusieurs méthodes de lutte contre ces hémiparasites avec les plus applicables que sont la lutte mécanique, la lutte biologique et la sélection de variétés résistantes. Il apparaît donc évident que la réussite de la promotion des chaînes de valeurs du karité dépend largement du contrôle des contraintes de cette filière parmi lesquelles se trouvent les Loranthaceae. Or, la connaissance du niveau d'infestation du karité par les Loranthaceae et des facteurs à l'origine de la variation de cette infestation peut contribuer à la performance des approches de lutte contre ces hémiparasites. C'est dans ce contexte que s'inscrit la présente étude qui vise à évaluer l'influence des facteurs écologiques sur la diversité et l'impact des Loranthaceae sur le karité au Nord du Bénin. 


\section{Matériel et Méthodes}

\section{Milieu d'étude}

Les études ont été conduites au Nord du Bénin (Figure 1) situé entre les latitudes $8^{\circ} 30^{\prime} \mathrm{N}$ et $12^{\circ} 25^{\prime} \mathrm{N}$ et les longitudes $0^{\circ} 45^{\prime} \mathrm{E}$ et $3^{\circ} 45^{\prime} \mathrm{E}$, et à cheval entre la zone de transition soudano-guinéenne et la zone soudanienne. La zone d'étude s'étend sur une superficie de $72597 \mathrm{~km}^{2}$ soit $63,26 \%$ de la superficie du Bénin. Deux types de climat y sont distingués : le climat de type tropical humide de transition caractérisé par deux ou quatre saisons avec une pluviosité annuelle variant de 1200 à $1300 \mathrm{~mm}$ et le climat de type tropical sec caractérisé par deux saisons avec une pluviosité annuelle comprise entre 900 et $1200 \mathrm{~mm}$ (Akoègninou et al., 2006). La végétation est caractérisée par des savanes soudaniennes parsemées de galeries forestières à Pterocarpus santalinoides, de forêts denses sèches et de forêts claires à Isoberlinia doka et Uapaca togoensis (Akoègninou, 2004).

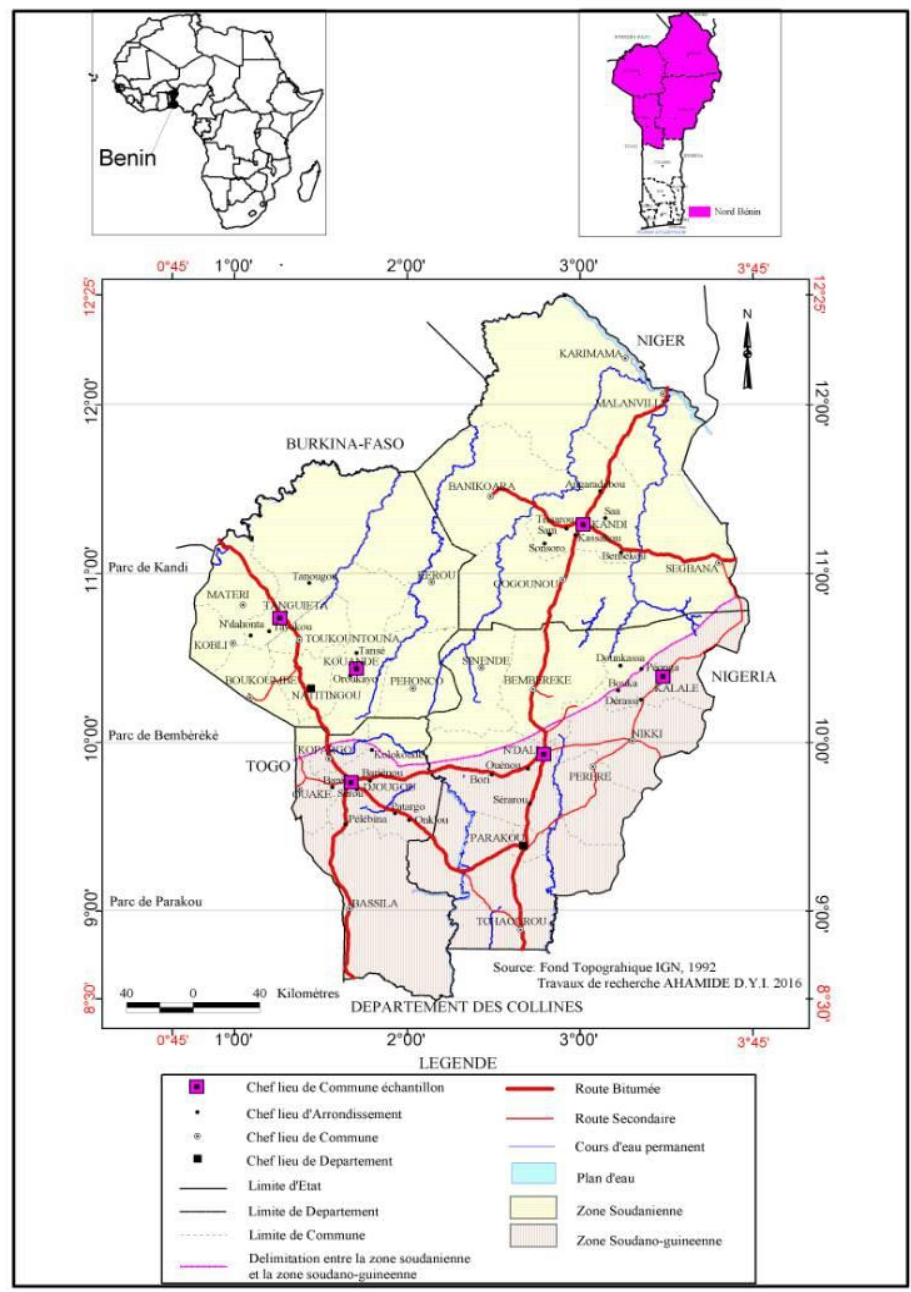

Figure 1 : Localisation du milieu d'étude au Nord du Bénin. 


\section{Méthode}

\section{Collecte de données}

La carte de distribution des parcs à karité du Bénin a été utilisée pour le choix des localités d'étude (Gnanglé et al., 2012). Elle a l'avantage de présenter un gradient climatique allant de la zone de transition soudanoguinéenne à la zone soudanienne du pays. Elle a permis d'identifier au nord du Bénin 3 parcs. Il s'agit des parcs de Parakou, de Bembèrèkè et de Kandi (Figure 1). Six communes ont été choisies en tenant de l'existence des zones phytogéographiques, de la densité des arbres à karité dans les localités, de l'ampleur de l'attaque des Loranthaceae au sein des vergers ainsi que de la présence d'aires protégées. Ce sont : Djougou, N'dali et Kalalé dans la zone soudano-guinéenne ; Kouandé, Kandi et Tanguiéta dans la zone soudanienne (Figure 1).

Pour les inventaires botaniques, la méthode de relevés de surface a été choisie (Hall et Swaine, 1981). Elle a consisté à poser 48 placeaux carrés de 1 ha équitablement répartis par commune puis entre les champs et les aires protégées constituées de forêts classées et de parcs nationaux. Le diamètre à hauteur de poitrine d'homme ou à $1,30 \mathrm{~m}$ du sol de chaque pied de karité adulte $(\mathrm{dbh} \geq 10 \mathrm{~cm})$ et le nombre de touffes de chaque espèce de Loranthaceae ont été recensés. Les échantillons de Loranthaceae ont été récoltés sur le terrain et l'identification des espèces a été faite à l'aide de la Flore Analytique du Bénin (Akoègninou et al., 2006).

\section{Traitement des données}

Les données collectées ont permis des calculer la diversité des Loranthace. Elle a été déterminée par le biais de la richesse spécifique qui est le nombre d'espèces de Loranthaceae. Par zone phytogéographique, par habitat et par pied de karité, cette densité a été évaluée. L'impact des Loranthaceae sur le karité a été aussi estimé. Les paramètres utilisés sont le taux d'infestation et la densité parasitaire. Le taux d'infestation (Ti) tel qu'utilisé par Houéhanou et al. (2011), nous a permis d'exprimer la proportion des pieds de karités parasités dans les zones. Il s'exprime en pourcentage et se calcule par la formule suivante :

$$
\mathrm{Ti}=\frac{\mathrm{NP}}{\mathrm{NT}} \times 100
$$

avec NP - nombre de pieds de karité parasités par placeau et NT nombre total de pieds de karité explorés par placeau. Des valeurs moyennes de ce taux ont été calculées par champ, aire protégée, zone phytogéographique et parc à karité. Le test de comparaison de deux moyennes a permis de comparer les valeurs moyennes obtenues.

La densité parasitaire est le nombre de touffes de Loranthaceae recensé. Elle a été évaluée par espèce parasite et sujet parasité en tenant 
compte des habitats (champ, aire protégée), des zones phytogéographiques des parcs à karité. La variation de la densité parasitaire en fonction des différents paramètres a été évaluée à l'aide de l'analyse de variance (ANOVA) grâce au logiciel R3.3.0. Une transformation logarithmique a été préalablement effectuée pour d'assurer la normalité des données obtenues.

L'évaluation de la densité parasitaire par pied de karité a permis de définir cinq niveaux d'infestation (Houéhanou et al., 2011) :

- N1: très faible infestation (1-5 touffes sur un pied);

- N2: faible infestation (6-10 touffes sur un pied);

- N3: infestation moyenne (11-15 touffes sur un pied);

- N4: infestation forte (16-20 touffes sur un pied);

- N5: infestation très forte (plus de 20 touffes sur un pied).

La proportion des pieds de karité parasités appartenant à chaque niveau d'infestation a été calculée par placeau suivant la formule : $\quad P=\frac{\mathbf{n i}}{\mathbf{N}}$ avec ni - nombre de pied de karité parasités appartenant au niveau i et $\mathrm{N}$ nombre total de pieds de karité parasités. Une analyse de variance a été effectuée pour comparer les niveaux d'infestations en fonction des dbh des pieds de karité parasités par habitat et par zone phytogéographique. Le coefficient de corrélation de Pearson et sa significativité ont permis d'établir le lien entre le niveau d'infestation et le dbh des pieds parasités.

\section{Résultats}

\section{Diversité des Loranthaceae parasites du karité}

Au total, 3 espèces de Loranthaceae ont été recensées sur le karité. Il s'agit de : Agelanthus dodoneifolius avec une densité moyenne de 191,75 \pm 140,56 touffes/ha. Ensuite vient Tapinanthus globiferus pour une densité de $70,57 \pm 69,58$ touffes/ha. T. ophiodes avec une densité de 2 touffes/ha est le parasite à plus faible densité dans la zone d'étude. Au regard des zones phytogéographiques et des habitats, Agelanthus dodoneifolius et Tapinanthus globiferus sont localisées partout tandis que $T$. ophiodes est seulement présente dans la zone soudanienne et dans les aires protégées. La richesse spécifique en parasite varie entre 1 et 2 espèces de Loranthaceae par pied de karité. Elle oscille entre 1 à 2 espèces dans les champs, tandis que dans les aires protégées, elle est de 1 à 3 espèces.

\section{Impact des Loranthaceae sur le karité}

\section{Taux d'infestation, densité parasitaire et niveau d'infestation}

Le taux moyen d'infestation obtenu varie significativement entre les habitats $(\mathrm{p}=0,00)$, mais, il reste stable entre les zones phytogéographiques $(\mathrm{p}=0,06)$ et dans les parcs à karité $(\mathrm{p}=0,42 ; \mathrm{p}=0,20)$ (Tableau 1). Ce taux est en effet de 87,25\% dans les champs contre 42,49\% dans les aires 
protégées. La densité parasitaire moyenne par pied de karité varie significativement entre les habitats $(\mathrm{p}=0,03)$ et les zones phytogéographiques $(\mathrm{p}=0,02)$, mais reste stable au sein des parcs à karité $(\mathrm{p}$ $=0,11 ; p=0,29)$. Elle est de $14,76 \pm 1,06$ touffes dans les champs contre $3,62 \pm 10,05$ touffes dans les aires protégées puis de $14,4 \pm 11,89$ touffes dans la zone soudanienne contre $7,71 \pm 3,18$ touffes dans la zone soudanoguinéenne.

Tableau 1 : Variation du taux d'infestation et de la densité parasitaire moyenne par pied de karité

\begin{tabular}{|c|c|c|c|c|}
\hline & $\begin{array}{c}\text { Taux } \\
\text { d'infestation } \\
(\%)\end{array}$ & Probabilité & $\begin{array}{c}\text { Densité } \\
\text { moyenne/pied } \\
\text { (touffes) }\end{array}$ & Probabilité \\
\hline \multicolumn{5}{|c|}{ Habitat } \\
\hline Champs & 87,25 & \multirow[t]{2}{*}{0,00} & $14,76 \pm 1,06$ & \multirow[t]{2}{*}{0,03} \\
\hline Aires Protégées & 42,39 & & $3,62 \pm 10,05$ & \\
\hline \multicolumn{5}{|c|}{ Zone phytogéographique } \\
\hline Soudanienne & 71,83 & \multirow[t]{2}{*}{0,06} & $14,40 \pm 11,89$ & \multirow[t]{2}{*}{0,02} \\
\hline $\begin{array}{l}\text { Soudano- } \\
\text { guinéenne }\end{array}$ & 65,86 & & $7,71 \pm 3,18$ & \\
\hline \multicolumn{5}{|l|}{ Parc à karité } \\
\hline Kandi & 70,49 & 0,42 & $10,07 \pm 6,03$ & \multirow[t]{2}{*}{0,11} \\
\hline Bembèrèkè & 73,43 & \multirow[t]{2}{*}{0,20} & $18,33 \pm 14,64$ & \\
\hline Parakou & 65,90 & & $7,71 \pm 3,18$ & 0,29 \\
\hline
\end{tabular}

La Figure 2 présente la variation en pourcentage des pieds de karité selon les niveaux d'infestation par habitat. Il y ressort que dans les aires protégées, la proportion des pieds de karité de très faible niveau d'infestation est plus élevée que celle enregistrée dans les champs. En ce qui concerne les pieds de karité de très fort niveau d'infestation, on note une proportion plus élevée dans les champs que celle obtenue dans les aires protégées. Cette tendance est la même lorsqu'on considère les différentes zones phytogéographiques (Figure 3) où la proportion des pieds ayant un très fort niveau d'infestation est plus importante en zone soudanienne que celle enregistrée en zone soudano-guinéenne. 


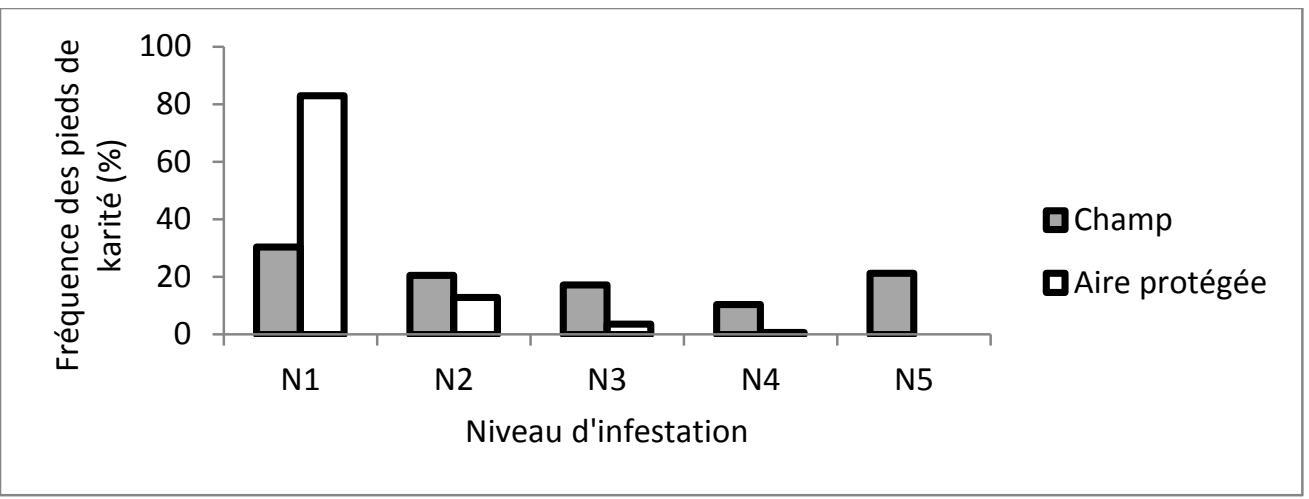

Figure 2 : Variation en pourcentage des pieds de karité en fonction du niveau d'infestation par habitat

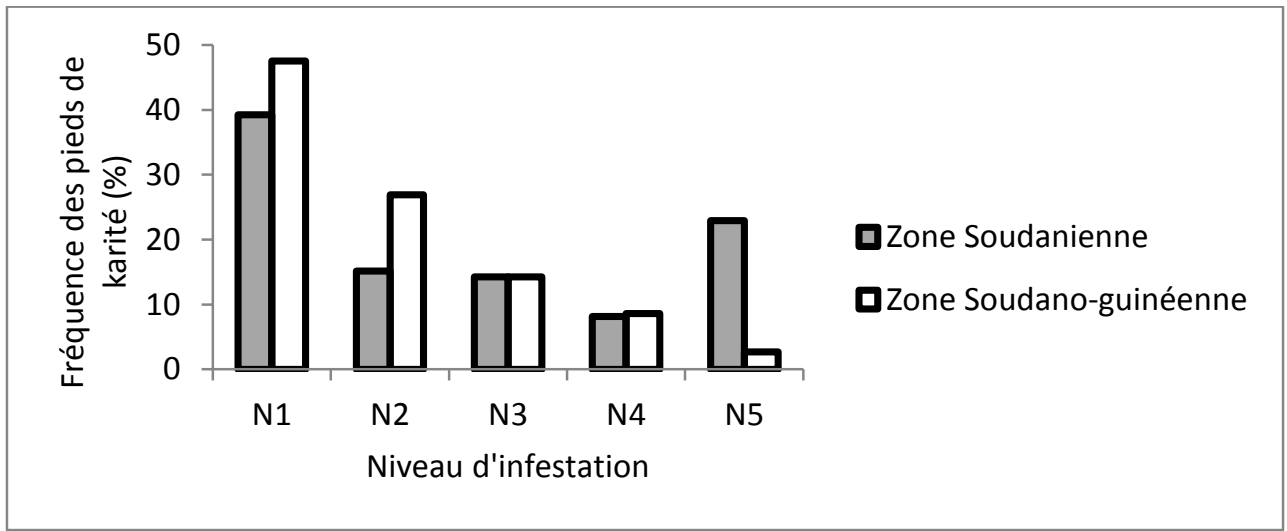

Figure 3 : Variation en pourcentage des pieds de karité en fonction du niveau d'infestation par zone phytogéographique.

\section{Diamètre des pieds de karité et niveau d'infestation}

En considérant les zones phytogéographiques, on note que dans les deux zones, suivant les valeurs moyennes des dbh, les pieds ayant les niveaux d'infestation les plus forts sont ceux ayant les dbh les plus élevés (Figure 4). En zone soudanienne, le dbh moyen des pieds de karité infestés est de $33,88 \mathrm{~cm}$ et le dbh minimum est de $10,19 \mathrm{~cm}$. Dans la zone soudanoguinéenne, le dbh moyen des pieds de karité infestés est de 35,26 cm et le dbh minimum est de $15,29 \mathrm{~cm}$.

Le coefficient de corrélation de Pearson entre le nombre de touffes de Loranthaceae et le dbh des pieds de karité parasités obtenu est significatif ( $\mathrm{p}$ $=0,00)$. Dans la zone soudanienne, ce coefficient est de 0,61 contre 0,46 en zone soudano-guinéenne. On relève que le nombre de touffes de Loranthacae augmente en fonction de la croissance du dbh des sujets parasités. De plus, la structure en diamètre du karité indique une différence hautement 
significative entre les niveaux d'infestation dans la zone soudanienne $(\mathrm{F}=$ $63,92 ; p=0,00)$ et dans la zone soudano-guinéenne $(F=13,23 ; p=0,00)$.

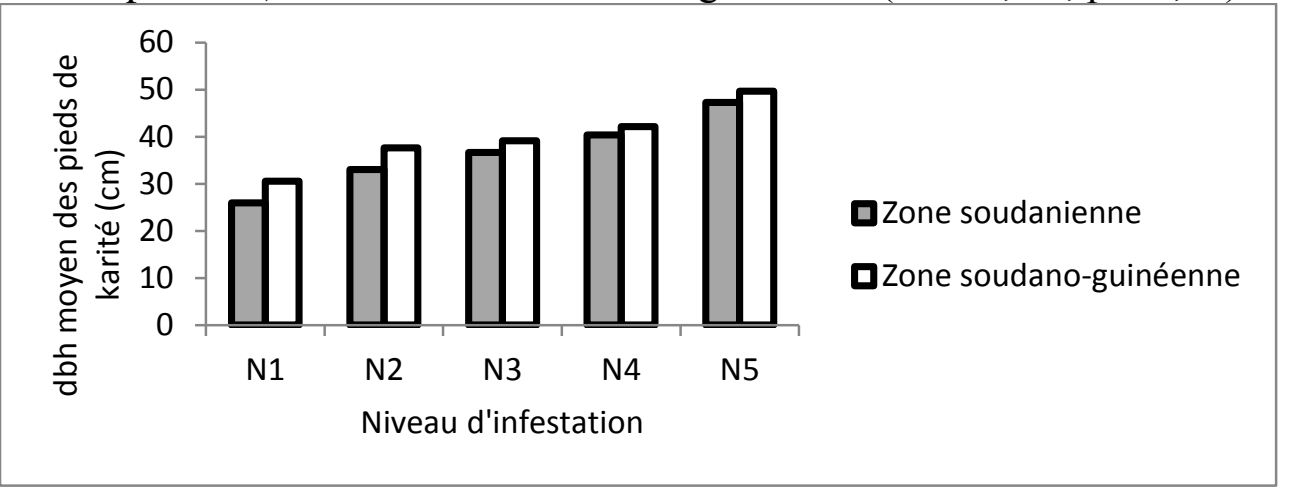

Figure 4 : Diamètre moyen des individus de karité en fonction du niveau d'infestation par zone phytogéographique.

Sur le plan de l'habitat du karité, les valeurs moyennes des dbh révèlent que les pieds ayant les dbh les plus élevés sont les plus infestés aussi bien dans les champs que dans les aires protégées (Figure 5). Dans les champs, le dbh moyen des individus infestés est de 36,44 cm et le minimum est de 10,51 cm. Dans les aires protégées, ce dbh moyen est de 27,66 cm et le minimum est de $10,19 \mathrm{~cm}$. Le coefficient de corrélation de Pearson entre le nombre de touffes de Loranthaceae et le dbh des sujets parasités est de 0,55 et 0,46 respectivement dans les champs et dans les aires protégées. La valeur du coefficient obtenue montre une augmentation du nombre de touffes de parasites au fur et à mesure que le dbh des pieds de karité infestés croit. Par ailleurs, il existe une différence hautement significative entre les niveaux d'infestation des pieds de karité en fonction du dbh dans les champs $(\mathrm{F}=$ $48,76 ; p=0,00)$ et celui des aires protégées $(F=6,41 ; p=0,00)$.

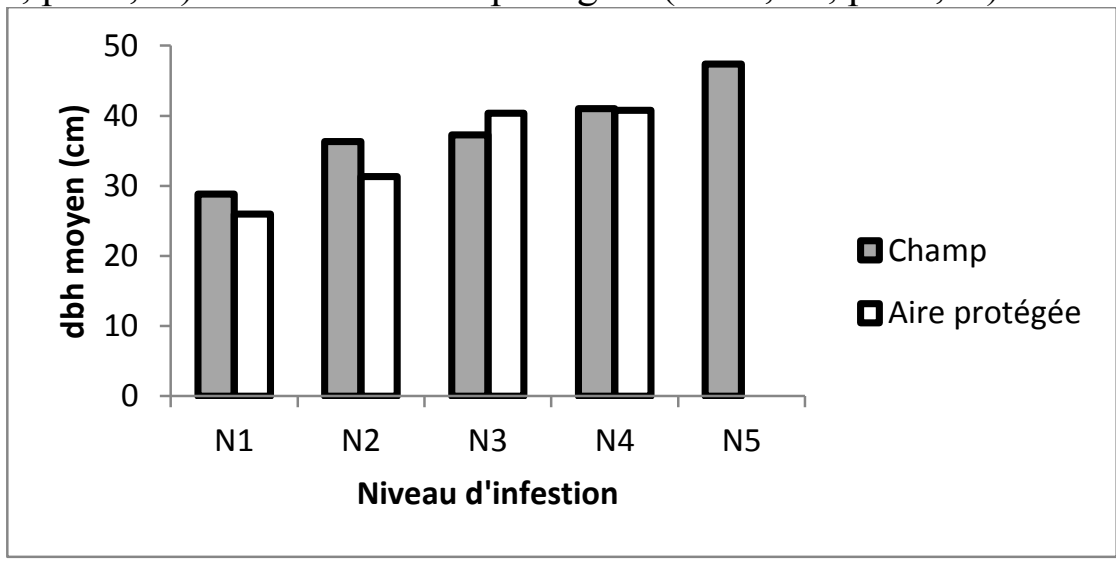

Figure 5 : Diamètre moyen des individus de karité et niveau d'infestation par habitat 


\section{Discussion}

Cette étude a permis d'inventorier trois espèces de Loranthaceae (Agelanthus dodoneifolius, Tapinanthus globiferus et T. ophiodes), parasites du karité, dans le Nord du Bénin. Selon Akoègninou et al. (2006), au Benin, la diversité floristique en Loranthaceae est de sept espèces dans la même zone. Ces résultats sont en accord avec ceux de Boussim et al. (1993) qui ont signalé la présence de ces mêmes espèces sur le karité au Burkina Faso. Cependant, le nombre d'espèces de Loranthaceae récoltées au cours de cette étude est inférieur à celui de 5 espèces de cinq espèces inventoriées dans les plantations d'agrumes au sud et au centre du Bénin (Houénon, 2012) et à celui de 6 espèces recensées par Ahamidé et al. (2015) sur Cola nitida au Sud du pays. La différence constatée dans le nombre d'espèces est due aux conditions climatiques. En effet, les conditions climatiques influencent la répartition des Loranthaceae. Les résultats ont montré qu'il existe une forte affinité entre le karité, Agelanthus dodoneifolius et Tapinanthus globiferus comme l'ont déjà signalée Boussim et al. (1993) au Burkina Faso. Ce qui n'est pas le cas au niveau des agrumes et du colatier. Pour les agrumes, leur affinité est plus forte avec $T$. globiferus (Houénon, 2012) tandis que $C$. nitida présente avec Phragmanthera capitata et Globimetula cupulata une forte affinité (Ahamidé et al., 2015).

Le taux d'infestation du karité est de $87 \%$ dans les champs et similaire à celui de $80 \%$ mentionné dans les parcs agroforestiers environnant le parc national de la Pendjari au Nord du Bénin (Houéhanou et al., 2011). Mais, il est inférieur à ceux de $95 \%$ obtenu par Boussim et al. (1993) au Burkina-Faso et $96 \%$ enregistré par Soro (2005) au Nord de la Côte d'Ivoire. Par contre, il supérieur à ceux de 60 et $53 \%$ rapportés respectivement par Houénon (2012) et Ahamidé et al. (2015) dans les plantations d'agrumes et celles de Cola nitida au Sud du Bénin. Ce taux est nettement supérieur à celui de 52,63\% enregistré sur le safourtier (Dacryodes edulis) par Massako et al. (2013) au Cameroun.

Les résultats ont montré que l'impact des Loranthaceae est plus sévère dans les champs que dans les aires protégées si nous considérons la valeur du taux d'infestation et le nombre moyen de touffes par pied de karité. Mais, cet impact ne varie pas en zones phytogéographiques et en considérant le taux d'infestation et le nombre moyen de touffes par pied de karité, dans les parcs à karité. La variation de l'impact des Loranthaceae sur le karité entre les champs et aires protégées relevée dans cette étude concorde avec les travaux de Houéhanou et al. (2011) réalisés dans le parc national de la Pendjari au Nord du Bénin. Ces auteurs rapportent que le karité des champs et jachères est plus parasité que celui des aires protégées. Cette différence peut s'expliquer par plusieurs facteurs. En considérant le système de reproduction et de propagation des Loranthaceae, selon Boussim et al. 
(1993), la dispersion des parasites sur le karité est assurée par les oiseaux disséminateurs du genre Pogoniulus. En effet, selon ces auteurs, ces oiseaux, pour une question de sécurité, fuyant la présence humaine, disséminent mieux les graines des parasites dans les champs que dans les aires protégées plus calme et moins bruyantes. D'où le fort niveau d'infestation relevé dans les champs par rapport aux aires protégées (Houéhanou et al., 2011).

Le faible niveau d'infestation du karité dans les aires protégées s'expliquerait par la forte diversité des ligneux qu'elles abritent. En effet, en cas d'une forte diversité d'hôtes, les oiseaux disséminateurs ont la possibilité de déposer une partie des graines de Loranthaceae sur des hôtes autres que le karité et parmi lesquels se trouvent des hôtes insensibles aux Loranthaceae.

Les résultats ont montré que la densité parasitaire des pieds de karité augmente au fur et à mesure que leur dbh croît. Ces résultats sont similaires à ceux obtenus par Houéhanou et al. (2011) sur le karité dans le parc national de la Pendjari, Ahamidé et al. (2015) sur Cola nitida au sud du pays et Edagbo et al. (2013) sur Irvingia gabonensis au Nigeria. Ceci est dû au fait que les arbres de plus gros diamètres sont probablement les plus âgés, les plus fréquentés par les oiseaux car, ils offrent une plus grande sécurité face aux rapaces (Traoré et al., 2003). L'état du karité et son niveau d'infestation peuvent également justifier le fort niveau d'infestation du karité dans les champs. En effet, les champs sont sujets à des pratiques agricoles telles que les labours, la réduction de la densité des arbres, la fertilisation du sol, le sarclage et la rareté des feux de végétation (Lamien, 2001; Kelly et al., 2007). Ces pratiques contribuent au développement des pieds de karité avec un accroissement de leur floraison et fructification (Djossa et al., 2008) qui sont des conditions favorables à l'installation, au développement et à la reproduction des Loranthaceae. Toutefois, il est à signaler que de façon synergique avec le dbh, d'autres paramètres tels que la hauteur et la position centrale ou périphérique des individus de $V$. paradoxa parasités expliqueraient mieux la variation de la densité parasitaire.

\section{Conclusion}

Les Loranthaceae, constituent une grave menace pour les arbres à karité. Il ressort de cette étude que le karité est infesté par trois espèces de Loranthaceae dans le Nord du Bénin. Agelanthus dodoneifolius et Tapinanthus globiferus sont les parasites les plus abondants sur le karité dans cette partie du pays. Le taux d'infestation du karité obtenu dans les champs est de $87 \%$ contre $42 \%$ enregistré dans les aires protégées. L'ampleur des attaques du karité par les plantes vasculaires parasites est importante. Il serait souhaitable de mettre en place des stratégie de lutte contre ces hémiparasites qui affectent la durabilité de ce fruitier en tenant compte des facteurs relatifs aux champs (présence humaine, faible diversité des ligneux, prépondérance 
des sujets à gros diamètres). Il conviendrait également que ces stratégies de lutte tiennent compte des usages des Loranthaceae parasites du karité dans la zone d'étude.

\section{References:}

1. Ahamidé DYI, Tossou MG, Adomou AC, Houénon GJ, Yédomonhan $\mathrm{H}$ et Akoègninou A : 2015. Diversité, impacts et usages des Loranthaceae parasites de Cola nitida (Vent.) Schott. \& Endl. au Sud-Bénin. Int. J. Biol. Chem. Sci., 9(6): 2859-2870.

2. Akoègninou A : 2004. Recherches botaniques et écologiques sur les forêts actuelles du Bénin. Thèse d'Etat. Université de CocodyAbidjan (Côte d'Ivoire). 326p.

3. Akoègninou A, van der Burg WJ et van der Maesen LJG : 2006. Flore Analytique du Bénin. Brackhuys Publishers:Wageningen, $1034 p$.

4. Bayala J, Teklehaimanot Z; Ouedraogo SJ: 2002. Millet production under pruned tree crowns in a parkland system in Burkina Faso. Agroforestry Systems, 54:203-214.

5. Boussim IJ, Guinko S, Sallé G : 1993. Tapinanthus parasite du karité au Burkina Faso. Bois et Forêts des Tropiques 238: 45-65.

6. Boussim IJ et Médah N : 2004. Méthodes de Lutte contre les Loranthaceae. Homme, plantes et environnement au Sahel occidental, 19:127-142.

7. Diarassouba N; Kouablan EK, Kanga AN, Van Dame P, Abdourahamane S : 2008. Connaissances locales et leur utilisation dans la gestion des parcs à karité en Côte d'Ivoire. Afrika focus, 21(1): 77-96.

8. Djossa BA, Fahr JT, Wiegand T, Ayihouenou BE, Kalko EK, Sinsin BA: 2008. Land use impact on Vitellaria paradoxa C.F. Gaerten. Stand structure and distribution patterns: a comparison of Biosphere Reserve of Pendjari in Atacora district in Benin. Agroforestry Systems 72: 205-220.

9. Edagbo DE, Ighere DA, Michael C: 2013. Mistletoe (Tapinanthus bangwensis) on the Conservation Status and Productivity of Irvingia gabonensis in Moor Plantation Area of Ibadan, Nigeria. Green. J. Agri. Sci., 3(10): 743-747.

10. Eyog Matig O, Gaoué OG, Dossou B, éditeurs : 2002. Réseau « Espèces Ligneuses Alimentaires ». Compte rendu de la première réunion du Réseau tenue 11-13 décembre 2000 au CNSF Ouagadougou, Burkina Faso. Institut International des Ressources Phytogénétiques. ISBN 92-9043-552-6. 241p. 
11. Gnanglè PC : 2005. Parcs à karité (Vitellaria paradoxa) (Gaertn. C. F.) (Sapotaceae) au Bénin: Importance socio-culturelle, caractérisations morphologique, structurale et régénération naturelle. Mémoire de DEA, Aménagement et Gestion des Ressources Naturelles, UAC/FSA, p.113.

12. Gnanglè PC, Egah J, Baco MN, Gbèmavo CDSJ, Kakaï GR et Sokpon N : 2012. Perceptions locales du changement climatique et mesures d'adaptation dans la gestion des parcs à karité au NordBénin. Int. J. Biol. Chem. Sci., 6(1): 136-149.

13. Hall JB et Swaine MD : 1981. Distribution and ecology of vascular plants in a rain forest vegetation in Ghana. Geobotany, $383 \mathrm{p}$.

14. Houéhanou TD, Kindomihou V, Sinsin B: 2011. Effectiveness of Conservation areas in protecting Shea trees against Hemiparasitic plants (Loranthaceae) in Benin,West Africa. Pl. Ecol. Evol., 144(3): 267-274.

15. Houénon GJ : 2012. Les Loranthaceae des Zones Guinéenne et Soudano-Guinéenne au Bénin et leur Impact sur les plantations Agrumicoles. Thèse de Doctorat, Université d'Abomey-Calavi, Abomey-Calavi, 133 p.

16. Kelly BA, Gourlet-Fleury S, Bouvet J-M : 2007. Impact of agroforestry practices on the flowering phenology of Vitellaria paradoxa in parklands in southern Mali. Agroforestry Systems 71:6775.

17. Lamien N : 2001. Organisation structurale et état sanitaire des populations fructifères de karité (Vitellaria paradoxa Gaertn.) de la région de Bondoukuy. Mémoire de DEA. Université de Ouagadougou, Burkina Faso.

18. Ministère de l'Agriculture de l'Elevage et de la Pêche (MAEP) : 2008. Atelier d'élaboration des stratégies des chaînes de valeur du karité au Bénin. Centre Guy Riobé de Parakou.

19. Massako F, Tchatat M, Mony R, Ladoh YCF, Dibong SD: 2013. Parasitisme de Dacryodes edulis par le genre Tapinanthus (Loranthaceae) et répartition de la Myrmécofaune associée à Logbessou Plateau (Douala, Cameroun). J. Appl. Biosci., 68: 53365348.

20. Sanou H, Lamien N : 2011. Vitellaria paradoxa, karité. Conservation et utilisation durable des ressources génétiques des espèces ligneuses alimentaires prioritaires de l'Afrique subsaharienne. Bioversity International (Rome, Italie). $12 \mathrm{p}$.

21. Soro D : 1999. Déparasitage par émondage et production en fruits du parc naturel à karité de Tengrela, dans le Nord de la Côte d'Ivoire. 
Mémoire de DEA d'Écologie Tropicale, Option Végétale, Université de Cocody, Abidjan, 84 p.

22. Soro K : 2005. Les Loranthaceae des légumineuses arborées introduites dans la zone forestière d'Oumé : espèces rencontrées, spectres d'hôtes et taux d'infestation. Mémoire de DEA, Université de Cocody, Abidjan, $51 \mathrm{p}$.

23. Soro K, Soro D, N'Guessan K, Gnahoua GM,Traoré D. 2010. Parasitisme des Loranthaceae sur les Hévéas en zone forestière des sous-préfectures de Gagnoa et d'Ouragahio, en Côte d'Ivoire. J. Anim. Plant Sci., 6(1): 597-604.

24. Traoré D, Da KP et Soro D. 2003. Lutte contre les plantes vasculaires parasites du karité, dans le Nord de la Côte d'Ivoire. Cas du parc naturel à karités de Tengrela, Universités de Cocody, Laboratoire de Botanique, Rapport de PEP/AISA-CI., 116p.

25. White F : 1983. The vegetation of Africa, a descriptive memoir to accompany the UNESCO/AETFAT/UNSO. Natural Resources Research 20: 1-356.

26. Wiesman Z, Maranz S : 2001. Chemical analysis of fruits of $V$. paradoxa. In : Teklehaimanot $\mathrm{Z}$ (Ed). Improved management of agroforestry parklands systems in Sub-Saharian Africa EU/INCO Project Contract IC18-CT98-0261, Third Annual Progress Report, University of Wales Bangor, Uk, 81-92. 